\title{
The association between experimental and clinical pain measures among persons with fibromyalgia and chronic fatigue syndrome
}

\author{
Michael E. Geisser ${ }^{\text {a,b,* }}$, Richard H. Gracely ${ }^{\text {a }}$, Thorsten Giesecke ${ }^{c}$, Frank W. Petzke ${ }^{c}$, \\ David A. Williams ${ }^{\text {a }}$, Daniel J. Clauw ${ }^{\text {a }}$ \\ ${ }^{a}$ Chronic Pain and Fatigue Research Center, Department of Internal Medicine, Division of Rheumatology, University of Michigan, \\ Ann Arbor, MI, United States \\ ${ }^{\mathrm{b}}$ Department of Physical Medicine and Rehabilitation, University of Michigan Health System, 325 E. Eisenhower Parkway, \\ Ann Arbor, MI 48108, United States \\ ${ }^{\mathrm{c}}$ Department of Anesthesiology and Intensive Care, University of Cologne, Cologne, Germany
}

Received 5 October 2005; received in revised form 30 January 2006; accepted 5 February 2006

Available online 20 March 2006

\begin{abstract}
Evoked or experimental pain is often used as a model for the study of clinical pain, yet there are little data regarding the relationship between the two. In addition, there are few data regarding the types of stimuli and stimulus intensities that are most closely related to clinical pain.

In this study, 36 subjects with fibromyalgia (FM), chronic fatigue syndrome (CFS), or both syndromes were administered measures of clinical pain and underwent a dolorimetry evaluation. Subjects also underwent experimental pain testing utilizing heat and pressure stimulation. Stimulation levels evoking low, moderate and high sensory intensity, and comparable levels of unpleasantness, were determined for both types of stimuli using random staircase methods. Clinical pain was assessed using visual analogue ratings and the short form of the McGill Pain Questionnaire (MPQ).

Ratings of heat pain sensation were not significantly associated with clinical pain ratings, with the exception of unpleasantness ratings at high stimulus intensities. Pain threshold and tolerance as assessed by dolorimetry were significantly associated with average measures of clinical pain. Both intensity and unpleasantness ratings of pressure delivered using random staircase methods were significantly associated with clinical pain at low, moderate and high levels, and the strength of the association was greater at increasingly noxious stimulus intensities.

These findings suggest that random pressure stimulation as an experimental pain model in these populations more closely reflects the clinical pain for these conditions. These findings merit consideration when designing experimental studies of clinical pain associated with FM and CFS.

(C) 2006 European Federation of Chapters of the International Association for the Study of Pain. Published by Elsevier Ltd. All rights reserved.
\end{abstract}

Keywords: Fibromyalgia; Chronic fatigue syndrome; Chronic pain; Experimental pain

\footnotetext{
${ }^{*}$ Corresponding author. Tel.: +1 734763 6501; fax: +1 734936 7048.

E-mail address: mgeisser@med.umich.edu (M.E. Geisser).
}

\section{Introduction}

Experimental studies designed to deliver noxious stimuli to subjects under controlled conditions are frequently used to make inferences about clinical pain conditions. Despite this, little is known about the 
relationship between experimental pain perception and clinical pain, and there is a lack of research on the experimental methods and types of stimuli that are most highly associated with clinical pain (Gracely, 1999). Such studies are highly important as evoked pain is increasingly being used to study central nervous system (CNS) abnormalities associated with clinical pain conditions such as fibromyalgia (FM), temporomandibular disorders, vulvodynia, and other entities (Geisser et al., 2003; Giesecke et al., 2004; Diatchenko et al., 2005; Petzke et al., 2003a). While pain is not a central feature of chronic fatigue syndrome (CFS), five of eight minor criteria (of which four are necessary to make the diagnosis) for CFS are pain-based (Fukuda et al., 1994). Common CNS abnormalities have been proposed to underlie all of these disorders (Clauw and Chrousos, 1997), and determining the experimental methods that best reproduce the clinical abnormalities associated with these conditions is crucial to their study.

Previous research has shown that persons with FM display heightened responsiveness to auditory tones (McDermid et al., 1996), contact thermal heat in both the noxious and innocuous ranges (Geisser et al., 2003; Kosek et al., 1996; Kosek and Hansson, 1997; Lautenbacher et al., 1994; Staud et al., 2001), ischemic pain (Kosek and Hansson, 1997), pressure applied to the thumb (Gracely et al., 2002; Petzke et al., 2003a) and electrical stimulation (Lautenbacher et al., 1994). Differences between FM subjects and controls have also been observed using methodologies that stimulate abnormal temporal summation of pain or wind-up (Staud et al., 2001, 2003) and the regulation of diffuse noxious inhibitory controls (Kosek and Hansson, 1997). However, only a few studies have examined how these abnormalities relate to the experience of clinical pain. Lautenbacher et al. (1994) reported low associations between measures of clinical pain and responses to electrocutaneous stimuli, pressure and heat. The authors also found that pressure pain thresholds at two sites were significantly associated with clinical pain. Staud et al. (2003) found that a combination of variables including measures of wind-up, pain-related negative affect, and tender point counts accounted for $49 \%$ of the variance in clinical pain. Further research is needed to determine the types of stimuli and experimental methods that are most highly associated with clinical pain states. In addition, previous research suggests that experimental methods that employ gradually ascending stimulation are more highly associated with psychological factors that may bias pain ratings (Petzke et al., 2003).

In the present study, we examined the relationships between clinical pain and a variety of evoked pain measures including gradually ascending pressure (dolorimetry) and a random staircase method of stimulus presentation of both pressure and heat stimuli. Based on prior research, we hypothesized that the random staircase methods would be more highly associated with measures of clinical pain compared to dolorimetry. In addition, we hypothesized that pressure pain perception would be more highly associated with measures of clinical pain compared to heat pain perception, as previous research has suggested that pressure sensitivity is highly associated with musculoskeletal pain syndromes (Diatchenko et al., 2005; Rollman and Lautenbacher, 2001). Since both momentary and average clinical pain were assessed, we also examined whether evoked pain was more highly associated with patients' usual pain, or more highly correlated with pain at the time of testing.

\section{Materials and methods}

\subsection{Subjects}

Thirty-six subjects who met either the 1990 American College of Rheumatology criteria for fibromyalgia (FM) (Wolfe et al., 1990), the diagnostic criteria for chronic fatigue syndrome (CFS) (Fukuda et al., 1994), or both diagnoses, were included in the study. Subjects with CFS had to have at least one pain symptom to be eligible. Eight subjects were diagnosed with FM alone, eight with CFS alone, and 20 fulfilled the diagnostic criteria for both disorders. Twenty-seven were female, and nine were male. Twenty-three were Caucasian, six were African-American, two were Hispanic, two were AsianAmerican, and three were of other descent. The mean age was $39.6(\mathrm{SD}=9.2)$ years. Mean duration of pain was 96.5 months $(\mathrm{SD}=80.9)$. Subjects with psychiatric disorders that did not interfere with study participation were not excluded.

The study was approved by the Georgetown University Medical Center's institutional review board, and informed consent was obtained from all participants for study on the General Clinical Research Center. All patients underwent a comprehensive screening during which the diagnosis was confirmed and co-morbidities were evaluated. Exclusion criteria were severe physical impairment, medical conditions that were capable of causing patients' symptoms (e.g., morbid obesity, autoimmune/inflammatory diseases, cardiopulmonary disorders), uncontrolled endocrine or allergic disorders (i.e., hyper-/hypothyroidism, diabetes, allergic rhinitis), malignancy, severe psychiatric illnesses (e.g., schizophrenia, substance abuse), factors known to affect the hypothalamic pituitary axis (HPA) or autonomic function (e.g., cigarette smoking, daily intake of caffeine exceeding the equivalence of two cups of coffee), or medication usage other than as-needed analgesics (excluding longterm narcotics). We did not exclude subjects with psychiatric conditions that are associated with HPA dysfunction (e.g., major depression). Eleven subjects who fulfilled the diagnostic criteria were excluded as 
these subjects did not complete all of the study measures examined in the current manuscript.

Subjects who qualified for inclusion in the study were scheduled for a 2-day study protocol. They were asked to discontinue intake of antidepressants up to four weeks ahead of the appointment (depending on the drug), but were allowed to use non-steroidal anti-inflammatory drugs until three days before the appointment. On the first day of the study, patients completed the self-report questionnaires and were familiarized with the pain testing paradigm. On the following day, they participated in a pain psychophysical testing session.

\subsection{Measures}

\subsubsection{Clinical pain}

Clinical pain was assessed using the short-form of the McGill Pain Questionnaire (MPQ; Melzack, 1987). This questionnaire contains 15 pain adjectives, and a total score is obtained by summing responses to all the items. The present pain intensity (PPI) subscale was examined as an indicator of pain intensity at the time of testing. The scale is sensitive to change produced by various pain interventions, and is highly correlated with the parent scale (Melzack, 1987).

Self-report of clinical pain intensity was also obtained by visual analogue scale (VAS) ratings. The scale was $100 \mathrm{~mm}$ long and anchored by the statements "no pain" on the left and "the most intense pain imaginable" on the right. Separate VAS scales were used to measure subjects' level of pain on the day of testing and average pain over the past month. VAS ratings have demonstrated good reliability (Boeckstyns and Backer, 1989; Revill et al., 1976) and concurrent validity when compared to other methods of pain measurement (Downie et al., 1978; Jensen et al., 1989).

\subsubsection{Pressure and heat pain assessment}

Evoked pain was assessed for both pressure and heat stimuli. Pressure pain sensitivity was evaluated by subjective scaling of pain sensations evoked by discrete 5-s pressure stimuli applied to the fixated left thumbnail with a $1-\mathrm{cm}^{2}$ hard rubber probe. Previous studies have shown that "neutral" regions, such as the thumb, accurately reflect an individual's overall pressure pain sensitivity (Petzke et al., 2001). The rubber probe was attached to a hydraulic piston, which was connected via a combination of valves to a second piston. Application of calibrated weights to the second piston produced controlled, repeatable pressure pain stimuli of rectangular waveform, that is, subjects experienced no pressure, then the target stimulus pressure when the appropriate weight was placed on the second piston. Subjects rated the intensity and unpleasantness dimensions of pressure pain sensations using a combined numerical (0-20) analog descriptor scale (Gracely et al., 1979). For each dimension, a series of 5-s stimuli were delivered to the right thumbnail in ascending order in $0.5 \mathrm{~kg}$ of force per square centimeter $\left(\mathrm{kg} / \mathrm{cm}^{2}\right)$ increments after an initial stimulus of $0.25 \mathrm{~kg} / \mathrm{cm}^{2}$, up to a maximum of $10 \mathrm{~kg} / \mathrm{cm}^{2}$. A second series of pressure stimuli was administered using the multiple random staircase (MRS) method (Gracely et al., 1988). A software system uses the data collected from the ascending series to compute starting stimulus intensities for another set of stimuli controlled by the method of MRS's. The MRS is an interactive system in which the software logic continuously adjusts the stimulus intensity to maintain ratings at several specific levels. In this implementation, three independent staircases are titrated to produce pain sensations rated between 0 and 1 (no sensation to faint pain), between 9 and 10 (mild-moderate pain), and between 13 and 14 (strong-slightly intense pain) on the $0-20$ box scale. In the remainder of this report, these levels are referred to as low, medium, and high. On each trial, the method randomly selects a staircase and delivers the stimulus intensity associated with that staircase. The response determines the next stimulus delivered by that staircase the next time it is selected. This determination is based on the previous response history and uses a dynamically changing step size to estimate the stimulus intensity required to produce the level of pain associated with each particular staircase. The method will deliver 12 stimuli for each of the three staircases, for a total of 36 stimuli delivered over $12 \mathrm{~min}$. If any staircase has not converged after 12 stimuli, the operator will be able to continue the method until convergence is reached or until 72 total stimuli have been delivered.

Heat pain sensations were evoked by a $1 \mathrm{~cm}$ diameter contact thermode system. A low-mass electrical heater on a water-perfused cold sink with feedback circuitry delivered precise stimulus waveforms with a ramp rate of $10^{\circ} \mathrm{C} / \mathrm{s}$. The thermal stimuli were delivered to the volar surface of the non-IV forearm. As with the pressure testing, both an ascending and a multiple random staircase series of thermal stimuli were presented to each subject. The temperatures required to evoke ratings of low, medium, and high pain intensity and unpleasantness were calculated for each subject.

\subsubsection{Dolorimetry}

A dolorimeter with a $1 \mathrm{~cm}^{2}$ tip was used to determine pain threshold and tolerance levels bilaterally at the thumb and lateral epicondyle. Pressure was increased at a rate of $1 \mathrm{~kg} / \mathrm{cm}^{2}$ per second and subjects were instructed to indicate when they first perceived pain (threshold) and when the pain became unbearable (tolerance). Pressure was stopped once the pain became unbearable or if $12 \mathrm{~kg} / \mathrm{cm}^{2}$ of pressure was reached. These sites were chosen as previous research has shown that these points are highly correlated with overall tenderness (Petzke et al., 2001). The measures from each 
side of the body were averaged to produce one value for each stimulus site.

\section{Results}

Table 1 shows the means and standard deviations for pressure and thermal intensities needed to evoke sensations of mild, medium, and high sensory intensity and unpleasantness using the random staircase procedure, and displays the threshold and tolerance averages for dolorimetry measured at the thumb and lateral epicondyle.

An initial analysis examined whether the patient groups differed on any of the clinical or experimental pain measures. Oneway analysis of variance (ANOVA) revealed that the groups significantly differed on VAS ratings of pain today $(F=7.0, p=.003)$ and pain over the past month $(F=4.1, p=.03)$. Post hoc tests (Duncan) indicated that subjects diagnosed with both FM and CFS had higher ratings of pain today compared to the other two groups, and had higher VAS ratings of pain over the past month compared to subjects diagnosed with CFS alone. In addition, the groups significantly differed on pain threshold $(F=3.2, p=.05)$ and tolerance $(F=3.2, p=.05)$ assessed by dolorimetry at the lateral epicondyle. Post hoc tests revealed that subjects with CFS alone had significantly higher pain threshold and tolerances compared to subjects with both CFS and FM.

Correlations between the clinical pain measures, dolorimetry, and heat and pressure pain measures (stimulus intensities needed to evoke different levels of pain sensation) are presented in Table 2. The correlations indicate that pressure stimuli delivered using the random staircase method were significantly associated with ratings on the MPQ for both unpleasantness and intensity

Table 1

Means (SD) of experimental heat and pressure measures

\begin{tabular}{lc}
\hline Measure & Mean (SD) \\
\hline Lateral epicondyle threshold $\left(\mathrm{kg} / \mathrm{cm}^{2}\right)$ & $5.4(2.5)$ \\
Lateral epicondyle tolerance $\left(\mathrm{kg} / \mathrm{cm}^{2}\right)$ & $7.2(3.0)$ \\
Thumb threshold $\left(\mathrm{kg} / \mathrm{cm}^{2}\right)$ & $6.6(3.0)$ \\
Thumb tolerance $\left(\mathrm{kg} / \mathrm{cm}^{2}\right)$ & $8.2(3.1)$ \\
Low pressure intensity $\left(\mathrm{kg} / \mathrm{cm}^{2}\right)$ & $2.3(1.8)$ \\
Medium pressure intensity $\left(\mathrm{kg} / \mathrm{cm}^{2}\right)$ & $4.7(2.5)$ \\
High pressure intensity $\left(\mathrm{kg} / \mathrm{cm}^{2}\right)$ & $6.5(2.7)$ \\
Low pressure unpleasantness $\left(\mathrm{kg} / \mathrm{cm}^{2}\right)$ & $2.7(2.3)$ \\
Medium pressure unpleasantness $\left(\mathrm{kg} / \mathrm{cm}^{2}\right)$ & $5.4(2.7)$ \\
High pressure unpleasantness $\left(\mathrm{kg} / \mathrm{cm}^{2}\right)$ & $7.4(2.9)$ \\
Low heat intensity $\left({ }^{\circ} \mathrm{C}\right)$ & $38.3(2.8)$ \\
Medium heat intensity $\left({ }^{\circ} \mathrm{C}\right)$ & $43.0(4.1)$ \\
High heat intensity $\left({ }^{\circ} \mathrm{C}\right)$ & $46.9(4.5)$ \\
Low heat unpleasantness $\left({ }^{\circ} \mathrm{C}\right)$ & $39.3(3.6)$ \\
Medium heat unpleasantness $\left({ }^{\circ} \mathrm{C}\right)$ & $44.9(4.8)$ \\
High heat unpleasantness $\left({ }^{\circ} \mathrm{C}\right)$ & $48.2(4.5)$ \\
\hline
\end{tabular}

Table 2

Correlations between experimental and clinical pain measures

\begin{tabular}{lllll}
\hline Measure & $\begin{array}{l}\text { McGill } \\
\text { total }\end{array}$ & $\begin{array}{l}\text { VAS } \\
\text { past } \\
\text { month }\end{array}$ & PPI & $\begin{array}{l}\text { VAS } \\
\text { today }\end{array}$ \\
\hline Dolorimeter & & & & \\
$\quad$ Lateral epicondyle threshold & $-.36^{*}$ & $-.34^{*}$ & -.17 & -.23 \\
Lateral epicondyle tolerance & $-.41^{*}$ & $-.35^{*}$ & -.24 & -.30 \\
Thumb threshold & -.22 & -.16 & -.09 & -.11 \\
Thumb tolerance & -.31 & -.25 & -.20 & -.24 \\
Pressure & & & & \\
Low intensity & $-.42^{*}$ & -.21 & -.18 & -.23 \\
Medium intensity & $-.48^{*}$ & -.23 & -.22 & -.24 \\
High intensity & $-.52^{*}$ & $-.33^{*}$ & -.27 & -.27 \\
Low unpleasantness & -.30 & .00 & -.13 & -.10 \\
Medium unpleasantness & $-.45^{*}$ & -.19 & -.22 & -.16 \\
High unpleasantness & $-.52^{*}$ & $-.35^{*}$ & -.27 & -.22 \\
Heat & & & & \\
Low intensity & -.14 & -.18 & .06 & -.11 \\
Medium intensity & -.20 & -.31 & .02 & -.17 \\
High intensity & -.24 & -.31 & -.03 & -.15 \\
Low unpleasantness & -.10 & -.07 & .03 & .00 \\
Medium unpleasantness & -.20 & -.24 & .01 & -.08 \\
High unpleasantness & $-.36^{*}$ & $-.35^{*}$ & -.15 & -.17 \\
\hline${ }^{*} p<.05$. & & & &
\end{tabular}

at all stimulus levels, with the exception of low unpleasantness ratings. In addition, pressure stimuli at high levels of intensity and unpleasantness were significantly associated with VAS ratings of pain over the past month. The magnitude of this association became greater as the stimulus intensity increased. Measures of pain threshold and tolerance assessed by dolorimetry at the lateral epicondle were significantly and inversely related to the MPQ total score and average VAS over the past month, indicating lower pain thresholds and tolerance were significantly associated with higher clinical pain. Pain thresholds and tolerances measured at the thumb using dolorimetry were not significantly associated with these same measures. None of the dolorimetry, heat or pressure pain measures were significantly correlated with measures of pain assessed on the day of testing.

Measures of heat pain sensitivity delivered using the random staircase procedure were not significantly associated with clinical pain ratings, with the exception of high unpleasantness ratings and McGill total pain scores and VAS ratings of pain over the past month.

To determine whether the significant correlations obtained between the experimental and clinical pain measures significantly differed across experimental methods, the formula for comparing two correlation coefficients from related samples was utilized (Weinberg and Goldberg, 1979, p. 412). Comparing the associations between intensity and unpleasantness levels and clinical pain assessed by the MPQ utilizing the MRS pressure method versus heat, the associations with 
MRS pressure were significantly higher for the medium $(t=-2.3, p=.03)$ and high $(t=-2.8, p=.01)$ intensity stimuli compared to the same levels obtained using MRS heat. A similar result was also obtained for medium $(t=-2.1, p=.05)$ unpleasantness stimuli. When VAS ratings of pain during the past month were examined, the associations between this measure and stimulation levels obtained using MRS heat and MRS pressure did not differ. The correlations between dolorimetry and clinical pain did not significantly differ from those observed between MRS heat or pressure and clinical pain. The magnitude of the associations between MRS pressure and clinical pain, and dolorimetry and clinical pain, were also not significantly different.

As trends were evident suggesting that higher stimulation levels were more strongly associated with clinical pain compared to less intense levels, these correlations were also compared using the same method noted above. For dolorimetry, associations between dolorimetry and clinical pain comparing the threshold and tolerance measures did not significantly differ. For MRS pressure and heat, the associations across different intensity rating levels also were not significantly different. For unpleasantness ratings, the association between pressure high unpleasantness and VAS ratings of pain over the past month was significantly greater than the association between pressure low unpleasantness and this same measure of clinical pain $(t=-2.8, p=.01)$. Also, the difference in the associations between pressure low unpleasantness and MPQ scores and high pressure unpleasantness and MPQ scores approached significance $(t-1.8, p=.08)$.

\section{Discussion}

Pain sensitivity determined by pressure stimulation using the multiple random staircase (MRS) procedure was significantly and inversely associated with average measures of clinical pain intensity, while heat was not. Comparing the magnitude of the associations, the correlations between MRS measures of pressure and clinical pain as assessed by the MPQ were significantly higher than those obtained between MRS heat and clinical pain. None of the experimental pain measures were significantly associated with measures of clinical pain assessed at the time of testing. These findings suggest that responses to evoked pressure pain in patients with FM and CFS can be generalized to patients' overall clinical condition, and that fluctuations in clinical pain that may occur during psychophysical testing do not significantly influence evoked pain responses. These findings also suggest that pressure stimulation as an experimental pain model among subjects with FM and CFS more closely reflects the average clinical pain associated with these conditions, and is consistent with other research suggesting that mechanical stimulation is an especially sensitive measure for the analysis of pathology associated with musculoskeletal pain (Diatchenko et al., 2005; Rollman and Lautenbacher, 2001).

In general, ratings given to higher stimulus intensities were more strongly associated with average ratings of clinical pain. These findings highlight the importance of evoked pain studies and provide further justification for the use of suprathreshold stimuli in experimental pain paradigms. The findings also suggest that experimental application of innocuous stimuli as a model for clinical pain may not be as generalizable to clinical pain conditions. In addition, the findings suggest that methods used to assess pain thresholds may not be as generalizable to clinical pain compared to studies employing suprathreshold methods of pain stimulation. This conclusion needs to be interpreted cautiously as significantly higher correlations between higher levels of experimental pain stimulation and clinical pain were only obtained for unpleasantness ratings and not intensity ratings, and this finding was only evident using the MRS pressure stimulation method. Further research examining the risk/benefit of noxious stimulus intensities in relation to the generalizability of the findings and subject burden would be beneficial.

Pressure stimulation using both dolorimetry and random staircase methods were both significantly associated with average measures of clinical pain. However, our previous research suggests that random staircase methods are less prone to biases associated with gradually ascending stimuli, and therefore are less likely to be influenced by affective states that frequently accompany pain, such as depression. In the present study, the magnitude of the associations between random staircase measures of pressure sensation and clinical pain as assessed by the McGill were somewhat higher than they were for dolorimetry, although this difference was not statistically significant. Further research is needed to determine the types of evoked pain models that most closely reflect the mechanisms underlying different clinical pain conditions.

It should be noted that the design of the present study is cross-sectional, and therefore no inferences can be made about causality. In addition, this study only examined a few of the experimental pain paradigms published in the literature, and therefore the findings cannot speak to the generalizability of other experimental methods to clinical pain, such as electrical stimulation. Third, the study examined patients with pain associated with FM and CFS, and the findings may not be generalizable to other clinical pain conditions. Fourth, this study examined the correlation between clinical pain intensity and experimental pain perception, and did not examine the ability of experimental methods to discriminate between persons with and without chronic pain. Such a comparison would also be beneficial in examining the validity of various methods of experimental pain. 


\section{Acknowledgements}

Funding for this study came in part from Department of the Army Grant DAMD-17002-0018 and Grant M01 RR-13297 from the National Center for Research Resources, National Institutes of Health.

\section{References}

Boeckstyns MEH, Backer M. Reliability and validity of the evaluation of pain in patients with total knee replacement. Pain 1989;38:29-33.

Clauw DJ, Chrousos GP. Chronic pain and fatigue syndromes:overlapping clinical and neuroendocrine features and potential pathogenic mechanisms. Neuroimmunomodulation 1997;4:134-53.

Diatchenko L, Slade GD, Nackley AG, Bhalang K, Sigurdsson A, Belfer I, et al. Genetic basis for individual variations in pain perception and the development of a chronic pain condition. Human Molecular Genetics 2005;14:135-43.

Downie WW, Leatham PA, Rhind VM, Wright V, Branco JA, Anderson JA. Studies with pain rating scales. Ann Rheum Dis 1978;37:378-81.

Fukuda K, Straus SE, Hickie I, Sharpe MC, Dobbins JG, Komaroff A The International Chronic Fatigue Syndrome Study Group. The chronic fatigue syndrome: a comprehensive approach to its definition and study. Ann Intern Med 1994;121:953-9.

Geisser ME, Casey KL, Brucksch CB, Ribbens CM, Appleton BB, Crofford LJ. Perception of noxious and innocuous heat stimulation among healthy women and women with fibromyalgia:association with mood, somatic focus, and catastrophizing. Pain 2003; 102:243-50.

Giesecke J, Reed BD, Haefner HK, Giesecke T, Clauw DJ, Gracely RH. Quantitative sensory testing in vulvodynia patients and increased peripheral pressure pain sensitivity. Obstetrics \& Gynecology 2004;104:126-33.

Gracely RH. Studies of pain in human subjects. In: Wall PD, Melzack R, editors. Textbook of pain. New York: Churchill Livingstone; 1999. p. 385-407.

Gracely RH, Dubner R, McGrath PA. Narcotic analgesia: fentanyl reduces the intensity but not the unpleasantness of painful tooth pulp sensations. Science 1979;203:1261-3.

Gracely RH, Lota L, Walter DJ, Dubner R. A multiple random staircase method of psychophysical pain assessment. Pain 1988;32:55-63.
Gracely RH, Petzke F, Wolf JM, Clauw DJ. Functional magnetic resonance imaging evidence of augmented pain processing in fibromyalgia. Arthritis Rheum 2002;46:1333-43.

Jensen MP, Karoly P, O'Riordan EF, Bland Jr F, Burns RS. The subjective experience of acute pain: an assessment of the utility of 10 indices. Clin J Pain 1989;5:153-9.

Kosek E, Eckholm J, Hansson P. Sensory dysfunction in fibromyalgia patients with implications for pathogenic mechanisms. Pain 1996;68:375-83.

Kosek E, Hansson P. Modulatory influence on somatosensory perception from vibration and heterotopic noxious conditioning (HNCS) fibromyalgia patients and healthy subjects. Pain 1997;70:41-51.

Lautenbacher S, Rollman GB, McCain GA. Multi-method assessment of experimental and clinical pain in patients with fibromyalgia. Pain 1994;59:45-53.

McDermid AJ, Rollman GB, McCain GA. Generalized hypervigilance in fibromyalgia: Evidence of perceptual amplification. Pain 1996;66:133-44.

Melzack R. The short-form McGill Pain Questionnaire. Pain 1987;30:191-7.

Petzke F, Khine A, Williams D, et al. Dolorimetry performed at 3 paired tender points highly predicts overall tenderness. J Rheumatol 2001;28:2568-9.

Petzke F, Clauw DJ, Ambrose K, Khine A, Gracely RH. Increased pain sensitivity in fibromyalgia: effects of stimulus type and mode of presentation. Pain 2003;105:403-13.

Petzke F, Gracely RH, Park KM, Ambrose K, Clauw DJ. What do tender points measure? Influence of distress on 4 measures of tenderness. J Rheum 2003;30:567-74.

Revill SI, Robinson JO, Rosen M, Hogg MIJ. The reliability of a linear analogue for evaluating pain. Anesthesia 1976;31:1191-8.

Rollman GB, Lautenbacher S. Sex differences in musculoskeletal pain. Clin J Pain 2001;17:20-4.

Staud R, Vierck CJ, Cannon RL, Mauderli AP, Price DD. Abnormal sensitization and temporal summation of second pain (wind-up) in patients with fibromyalgia syndrome. Pain 2001;91:165-75.

Staud R, Cannon RC, Mauderli AP, Robinson ME, Price DD, Vierck Jr CJ. Temporal summation of pain from mechanical stimulation of muscle tissue in normal controls and subjects with fibromyalgia syndrome. Pain 2003;102:87-95.

Weinberg SL, Goldberg KP. Basic statistics for education and the behavioral sciences. Boston, MA: Houghton Mifflin Company; 1979.

Wolfe FW, Smythe HA, Yunas MB, Bennett RM, Bombardier C, Goldenberg DL, et al. The American College of Rheumatology 1990 criteria for the classification of fibromyalgia. Arthritis Rheum 1990;33:160-72. 